\title{
La desigualdad de ingresos y su incidencia en la participación electoral venezolana
}

\author{
Pérez López, Renato* \\ *Universidad Técnica de Cotopaxi \\ Latacunga, Ecuador \\ Correo electrónico: renato.perez@utc.edu.ec
}

Recibido: 08 mayo de 2019 Aprobado: 21 junio de 2019

\begin{abstract}
Resumen
El presente artículo tiene como objetivo analizar la relación entre participación electoral y desigualdad. Para esto, se construyó como medida de desigualdad el índice de Gini a nivel municipal y se estimó la participación ciudadana a partir de los niveles de abstención reportados por el Consejo Nacional Electoral en comicios presidenciales de 1998 y 2012, utilizando como unidad de análisis los 335 municipios del país. Se encontró evidencia de que mayores niveles de abstención electoral se asocian con mayores niveles de desigualdad de ingresos intramunicipal.
\end{abstract}

Palabras clave: Capital social, Desarrollo, Desigualdad, Ingresos, Participación electoral. 


\title{
Income inequality and its impact on the Venezuelan electoral participation
}

\begin{abstract}
This paper aims to assess the relationship between voter turnout and inequality. On one hand, a Gini coefficient was constructed on a municipal level as a measure of inequality. On the other hand, the abstention rate reported by the National Election Commission on the 1998 and 2012 presidential elections was used to represent civil participation using data from Venezuela's 335 municipalities. Evidence was found that higher levels of electoral abstention are associated with higher levels of intra-municipal income inequality.
\end{abstract}

Keywords: Social capital, Development, Inequality, Income, Electoral participation.

\section{Introducción}

Salamanca (2012), en su libro "Por qué vota la gente" concluye que, desde los inicios del período democrático hasta la década de los ochenta, Venezuela se caracterizó por tener altos niveles de participación electoral debido a los altos niveles de identificación con los partidos y niveles de legitimidad del sistema. Tal como apunta el autor: "...El elemento predictor fundamental del voto en Venezuela fue la identificación con un partido hasta los años 90 del siglo XX" (p.90). Luego de este período las razones por las que una persona decide participar en una elección no son tan evidentes.

Como todo fenómeno social, existen múltiples variables que pueden afectar la participación ciudadana en un país. En Venezuela se puede intuir que la reducción de la desigualdad de ingresos en los últimos diecisiete años pudo haber incidido en los niveles de participación, como consecuencia de las políticas implementadas con orientación a la redistribución progresiva de la riqueza.

En el marco de la participación social, es importante recalcar el papel que desempeñan los procesos electorales en revelar la propensión de los ciudadanos a involucrarse en actividades políticas, las cuales legitiman las gestiones de gobierno y benefician a la sociedad en conjunto, a través de su desarrollo, crecimiento institucional y económico. Fukuyama (1995) y Putnam (1993) responsabilizan de este fenómeno al crecimiento del capital social, como el canal que crea valor tanto individual como colectivo, mediante el fortalecimiento de un conjunto de redes y normas de reciprocidad de la sociedad afiliadas a ellas.

Siguiendo a Pickett \& Wilkinson (2010), la desigualdad deteriora el capital social en una sociedad, ya que crea situaciones desfavorables para la comunicación y colaboración entre individuos, al generar desconfianza no solo entre personas, sino también entre personas e instituciones, lo que se traduce finalmente en reducción de los niveles de participación social.

Banfield (1958), resalta cómo la falta de asociación política y organización corporativa, son un factor limitante importante en el desarrollo económico de los países que generan barreras para el progreso político. El autor estudia el comportamiento de una población al sur de Italia -con deficiencias estructurales de desarrollo institucional y económico- $\mathrm{y}$ concluye que en sociedades poco propensas a asociarse en grupos y participar en actividades políticas y sociales como esa, nadie trabaja 
para el beneficio de la comunidad a menos que le favorezca de alguna forma a sus intereses personales. Consecuentemente, la fragmentada participación ciudadana no genera conexiones entre principios políticos abstractos o ideologías y el comportamiento en concreto de las interrelaciones ordinarias del día a día.

El estudio de Banfield (1958) antes referido, subraya cómo en comunidades con pocos incentivos a la participación escasean figuras de liderazgo e iniciativas emprendedoras que fomenten el crecimiento y la confianza de la economía. Por ello, en sociedades con baja participación, poca movilidad social y estructuras institucionales pobres, hay débiles maquinarias electorales de partidos que fomenten la actividad ciudadana. De esta forma, Banfield, confirma la fuerte influencia que ejercen variables determinantes del capital social -como la asociación y la participación- sobre el desarrollo y crecimiento económico y social de una población.

En una nota muy similar, Putnam (2000) destaca la poderosa influencia que tienen las normas y redes de participación ciudadana sobre la calidad de la vida pública y el desempeño de las instituciones. En especial, el autor expone la vital importancia que tienen las redes en la sociedad como motores del desarrollo económico tanto para países industrializados como para aquellos en desarrollo. Los casos exitosos del Capitalismo Globalizado de Redes del Este Asiático y los Distritos Industrializados basados en redes de colaboración entre trabajadores y pequeños emprendedores de economías latinoamericanas, son claros ejemplos que destaca el autor.
Parte del mecanismo mediante el cual las normas y redes de participación ciudadana afectan al desarrollo económico de los países, es a través del rendimiento del gobierno representativo, donde procesos electorales y membresías en asociaciones ciudadanas definen la calidad y los estándares de gobernanza de las naciones. Es así como Putnam (2000) explica el rol de las redes organizadas y la participación cívica sobre la modernización socioeconómica y su estrecha vinculación con los conceptos de confianza social y capital social. Su relevancia radica en la capacidad de facilitar la coordinación y comunicación entre personas e instituciones, permitiendo resolver dilemas de acción colectiva.

Más adelante, Putnam (2000) toma como referencia la participación electoral cuando evalúa los comportamientos del compromiso civil, al considerarla el acto más sublime y sencillo de ciudadanía activa, cuyas tendencias se asemejan a aquellas de participaciones ciudadanas tanto a niveles locales como estadales. La evidencia ha demostrado que en las conexiones sociales y acciones cívicas, todas las formas de capital social están coherentemente relacionadas entre individuos, donde aquellos que son miembros de asociaciones tienen mayor propensión a participar en actividades políticas y sociales. El autor lleva a cabo la misma aseveración tanto a nivel individual entre personas como a nivel agregado entre países, donde demuestra fuertes correlaciones entre las membresías de asociaciones y la confianza social.

Gracias a colaboraciones como las de Banfield (1958) y Putnam (2000) se logra destacar el rol protagónico que desempeña la 
participación ciudadana -en especial aquella llevada a cabo en procesos electorales- como representante del capital social y motor de crecimiento y desarrollo de la sociedad en su conjunto. La importancia de estudiar cómo se alimentan los índices de participación cívica radica en los beneficios directos que genera sobre las economías y sociedades de los países, por lo que se recalca la pertinencia de revelar los efectos que tiene la desigualdad social sobre la participación ciudadana en una nación como la venezolana.

El siguiente escrito está organizado de la siguiente manera. En la sección 2 se hará una revisión de los conceptos claves para el entendimiento del presente artículo. La sección 3 describirá la metodología y los datos utilizados. En la sección 4 se presentará la evidencia econométrica. Por último se presentarán las conclusiones.

\section{Consideraciones teóricas}

\section{Desigualdad}

Comenzando con el desarrollo del concepto de desigualdad, merece destacar que el mismo no es sinónimo de diferencia. Según Phelan, M. \& Fundación Escuela de Gerencia Social (2006) diferencia se refiere a una o varias características y atributos que distinguen a personas y a grupos entre sí, como elementos culturales, lingüísticos, étnicos y demás. Por su parte la desigualdad "remite a las brechas o a las distancias que se establecen entre personas o grupos sociales, definidas a partir del acceso o distribución inequitativo de bienes, tanto en el tipo como en la cantidad"(p.2).
Por su parte, el concepto de desigualdad de ingresos enmarcado dentro del concepto de desigualdad social, se define como "una situación socioeconómica (no necesariamente vinculada con la apropiación o usurpación privada de bienes, recursos y recompensas), en un contexto de competencia y lucha" (p.113) (Silva, 2010). López-Aranguren (2005) por su parte describe la desigualdad social como la condición por la cual las personas tienen un acceso desigual a los recursos de todo tipo, a los servicios y a las posiciones que valora la sociedad.

Podemos así considerar la desigualdad social como una condición que aleja los agentes de una comunidad entre sí, dado un acceso inequitativo a recursos y servicios, debido a diferencias sexuales, religiosas, étnicas, económicas, políticas u otras. Por lo antes expresado el concepto de desigualdad contiene una amplia gama de enfoques, como pueden ser: las desigualdades sociales, económicas, jurídicas y educativas.

De esta forma se puede abordar puntualmente el término de desigualdad de ingresos como todas las disparidades en el acceso y distribución de bienes e ingresos económicos, en especial la distribución de la renta. El término se refiere normalmente a la desigualdad entre individuos y grupos al interior de una sociedad, pero también se puede referir a la desigualdad entre países y/o regiones.

\section{Medidas de Desigualdad}

La medida más utilizada para calcular los niveles de desigualdad de ingresos de una ciudad es el coeficiente de Gini; sin embargo debemos aclarar que no representa la única forma de cálculo. 
Goerlich (1998), plantea que existen distintas maneras o indicadores que miden la desigualdad, además del índice de Gini. El investigador destaca estudios de Theil (1967) quien propone dos medidas diferentes de desigualdad a partir del concepto de entropía de la teoría de la información. Los índices de Theil son "resultado de casos particulares de las clases de medidas generalizadas de entropía, conocidos como la familia de los índices de Theil” (p.18) (Goerlich, 1998).

Siguiendo a Goerlich (1998) el índice de Atkinson "define la renta igualitaria equivalente como aquel nivel de renta per cápita tal que, si fuese disfrutado por toda la población, generaría el mismo nivel de bienestar que la distribución inicial de la renta." (p.21). Esto significa que la función de bienestar social sería cóncava, recogiendo la pérdida de bienestar social asociada a una distribución desigual de la renta.

Goerlich (1998) concluye afirmando la preponderancia en el uso del índice de Gini como aquel indicador que goza mayor popularidad y aceptación debido a la facilidad de su uso y pragmática explicación de la desigualdad de ingresos en cualquier comunidad.

\section{Participación y abstención}

Como segunda variable fundamental en este trabajo, se debe aclarar qué se entiende por participación electoral, sus elementos más característicos, los mecanismos para medirla y su conexión con el capital social y la participación ciudadana. Como referencia inicial, se puede acotar la definición de participación electoral al proceso mediante el cual un grupo de personas votan en unas elecciones, siendo la abstención electoral su espejo, donde un grupo de personas decide no votar o simplemente no puede participar en unas elecciones.

Vale la pena remitirse al estudio de "Raíces de la abstención electoral", Domènech, (2011) evalúa los diversos motivos por los cuales las personas votan o se abstienen, pudiendo ser estos: instrumentales, utilitarios y consecuencialistas por un lado, o estructurales, morales e incrustados en un marco de acción colectiva por el otro.

Domènech se encarga de destacar varios estudios que abogan por la preponderancia de la motivación/desmotivación a votar como parte de "una participación individual más o menos sólidamente incrustada en un marco de acción colectiva" (p.1), donde los individuos van a votar o se abstienen de hacerlo por la sencilla razón de formar parte de un grupo social con intereses en común, encarnándose en asociaciones de personas afines entre sí.

Por su parte, Salamanca (2012) concluye que el acto de sufragar de los ciudadanos está condicionado por un conjunto de factores de carácter institucional, económico, social y político. Estos factores los divide principalmente en tres categorías. La primera, factores estructurantes, identificando como principales los aspectos culturales, sociales y sociodemográficos. La segunda, componentes desencadenantes, vinculados a la dinámica política y contextual, generando interés en participar. La última categoría son los cristalizadores, donde su eje central es la campaña política.

Es importante resaltar como se aborda el término de participación electoral enmarcado 
dentro de la participación política y ciudadana, a su vez como el referente más importante del capital social. En Venezuela Pérez (2006) explica por qué resulta que la participación ciudadana a través del sufragio representa la forma más extendida y frecuente de la participación política. Este argumento lo ratifica Briceño (2009) por lo incorporada que está la cultura electoral en el imaginario político del venezolano. Por su parte Pérez (2006) citando a Kim, et al. (1978) agrega que las elecciones representan la actividad participativa más utilizada en las democracias contemporáneas, "puesto que constituye la manera menos complicada de ejercer presión sobre la política gubernamental, requiere bajos niveles de información y poca iniciativa.”(p.75)

Pérez (2006) define la participación electoral como el número o porcentaje de ciudadanos que acudieron a las urnas de votación en elecciones políticas. Expone cómo su medición puede llevarse a cabo en términos agregados con los resultados de las votaciones, proveyendo información referente a las circunscripciones definidas políticamente y relacionadas con variables demográficas, económicas y culturales, o a nivel individual mediante encuestas que expliquen variables actitudinales, afectivas o de comportamiento, relacionadas con el contexto político, social y cultural.

Para el caso de Venezuela, Pérez (2006) destaca un significativo obstáculo para el análisis de la abstención como medidor de la participación electoral. Básicamente la abstención oficial es calculada en base al número de inscritos en el Registro Electoral Permanente (REP) venezolano, el cual es un registro voluntario que debería realizar el elector al cumplir los 18 años. El problema radica en que una parte de la población no determinada con exactitud, falla en el cumplimiento de esta formalidad o deber, quedando fuera de los registros oficiales de votación, es decir, hay una discrepancia entre el total de personas que se pueden inscribir en el REP y las que efectivamente lo hacen. El universo de personas posibles se conoce como Población en edad electoral. Adicionalmente, las deficiencias del Registro en su actualización como exclusión de fallecidos e inhabilitados políticamente, plantean serias dudas sobre su exactitud.

\section{Hechos estilizados}

Resulta interesante hacer visualización sobre la evolución de las principales variables de estudio, en particular la variación del coeficiente de Gini a nivel municipal y los niveles de abstención en las elecciones presidenciales de 1998 y 2012. Poder cuantificar la relación y dirección entre las variables otorga importantes oportunidades para el desarrollo de la sociedad.

El siguiente gráfico muestra las variaciones de los niveles de abstención en cada territorio. La intensidad del color rojo hace referencia a la mayor variación del indicador. Esta diferenciación se muestra a continuación:

$$
X=\frac{(a b s 2012-a b s 1998)}{a b s 1998}
$$

Una disminución de los niveles de abstención en el tiempo, arrojarían valores negativos en " $X$ ". Gráficamente, mientras más haya descendido la abstención, mayor intensidad del color rojo reportará el estado lo que indica mayores niveles de participación, y mientras 
menos negativo sea el indicador, el color rojo irá diluyéndose hasta ser blanco.

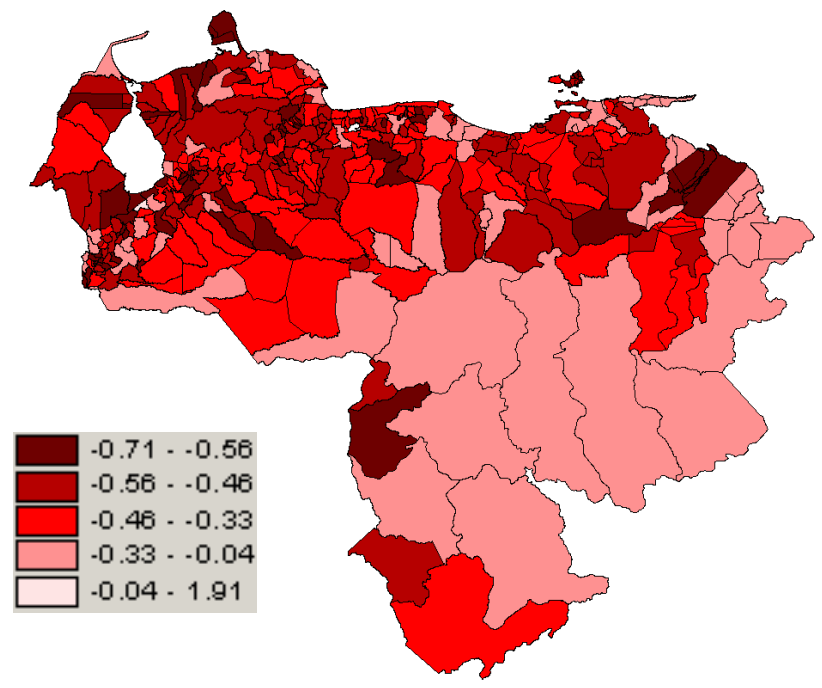

Figura 1. Variación de los niveles de abstención nivel municipal

Fuente: Instituto Nacional de Estadística INE y cálculos propios.

Elaboración: autor

Como conclusión, los niveles de abstención se redujeron en las elecciones presidenciales de 2012 con respecto a 1998.

Con relación al coeficiente de Gini, el siguiente gráfico muestra las variaciones de las desigualdades en cada territorio. La intensidad del color rojo hace referencia a la mayor variación del indicador. Esta diferenciación se muestra a continuación:

$$
X=\frac{(\text { gini2011 }- \text { gini2001) }}{\text { gini } 2001}
$$

Analizando la expresión, si la sociedad a nivel municipal se ha vuelto más igualitaria a nivel de ingresos entre 2001 y 2011, el indicador arrojará valores negativos. Haciendo la evaluación del indicador resultante en el gráfico, se tiene un mapa de Venezuela dividido territorialmente en municipios, mayormente de color rojo y bastante intenso. En efecto, las desigualdades de ingresos a nivel municipal se han reducido significativamente.

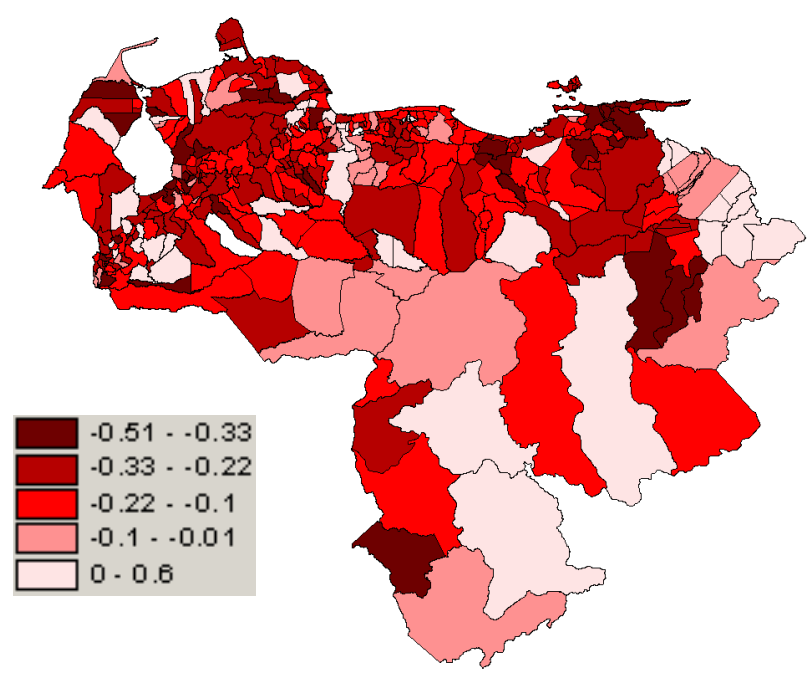

Figura 2. Variación del coeficiente de Gini a nivel municipal

Fuente: Instituto Nacional de Estadística INE y cálculos propios.

Elaboración: autor

$\mathrm{Al}$ analizar gráficamente ambas variables de interés, pareciera que la hipótesis planteada tiene sentido, que efectivamente hay una relación entre variables. Sin embargo, este análisis gráfico no permite saber cuál es la dirección en la relación de las variables, resultando necesario un análisis estadístico/econométrico.

\section{Materiales y métodos}

Los datos a utilizar para la variable dependiente fueron la abstención electoral de las elecciones presidenciales de 1998 (ABS_P_98) y 2012 (ABS_P_12) a nivel municipal. Esta variable fue extraída directamente de la página web del Consejo Nacional Electoral (CNE). 
Como principal variable independiente, se trabajó con un índice de Gini a nivel municipal estimado por el autor. Para la elaboración de este índice se utilizaron los ingresos declarados por los particulares durante el Censo de Población y Vivienda de 2001 (GINIBANDAS) y 2011 (GINIBANDAS_11). Por último para controlar el modelo, se decidió estudiar la influencia de los ingresos medios de los hogares por municipio con base del año 1997 sobre los niveles de abstención y desigualdad. Para el año 2001 la variable se denomina (ING_HOG_BASE97) y para el año 2011 (ING_BASE_97). El control probó ser significativo en ambos casos al 1\% de confianza, con un efecto sobre ambas variables en la dirección esperada. De esa forma, los ingresos medios mostraron tener una relación inversa a la abstención electoral, entendiendo que mientras mayores sean los ingresos de cada municipio, los niveles abstención se reducen.

Seguidamente, se incorporó una nueva variable que controlara el efecto que los ingresos medios de los hogares elevados al cuadrado tenían sobre los niveles de abstención. Para el año 2001 esta variable se denominó (ING_MEDIO_ CUAD) y para el año 2011 (INGMEDIO CUADRADO_2011). Así se evaluó a qué tasa podía incrementar la participación electoral a medida que se elevaban los ingresos medios de los hogares. Resultó que la variable tuvo un efecto positivo para las elecciones de 1998, indicando que la participación electoral se incrementa a tasa decreciente a medida que se elevan los ingresos medios de los hogares. Para los comicios electorales de 2012 ocurrió lo contrario, indicando que la participación electoral se incrementa a tasa creciente a medida que se elevan los ingresos medios de los hogares. Esto es un claro reflejo de cómo los incentivos económicos (medidos a partir del ingreso) han variado en el tiempo.

Y como última variable de control se decidió incorporar un índice de urbanización, entendiendo que municipios más urbanizados tenderían a ser más participativos por un tema de información disponible. Para el año 2001 esta variable se denominó (INDICE_URB) y para el año 2011 (INDICE_URB_2011). Dicho control demostró ser significativo y con la dirección esperada en ambos periodos electorales.

Para corregir el problema de endogeneidad, se probaron distintas variables para ser utilizadas como variable instrumental. La que mejor respondió como corrector del problema de endogeneidad fue la estructura poblacional activa (ESTRUCT_POB_ACT). La intuición para el uso de esta variable como instrumento es que, se crean desigualdades a nivel de ingresos entre las personas que están actualmente trabajando y las que no, lo que puede impulsar a las personas a participar electoralmente en búsqueda de que se hagan políticas que disminuyan esta brecha. De esta manera se estaría probando la dirección y el sentido de la hipótesis.

El modelo utilizado para estimar la incidencia de la desigualdad de ingresos sobre la participación electoral fue un modelo de regresión linear, llamado Mínimos Cuadrados Ordinarios (MCO)

La selección de este modelo se realizó en base al tipo de datos de la variable dependiente, ya que, al ser una variable continua, se partió del supuesto que sigue una distribución normal. 
Con respecto a las variables institucionales, se considera que el sistema democrático y la forma de elegir los gobernantes han sido constantes en el tiempo, es decir, las instituciones no han cambiado en ambos períodos. Las modificaciones sufridas en el sistema son del tipo operativos-tecnológicos (nombre del ente responsable, períodos de ejercicio, etc) que no alteran la condición del voto (uninominal, secreto, etc).

\section{Resultados y discusión}

El siguiente cuadro muestra los resultados obtenidos para el año 2001.

\section{Tabla 1}

\section{Modelo para 1998}

\begin{tabular}{|c|c|c|c|c|c|c|c|}
\hline Source & ss & df & \multicolumn{2}{|c|}{ MS } & & \multirow{3}{*}{$\begin{array}{l}\text { Number of obs }= \\
F(4,330)= \\
\text { Prob }>F\end{array}$} & 335 \\
\hline & & & & & & & 19.65 \\
\hline Model & .241587483 & 4 & .06039 & 871 & & & 0.0000 \\
\hline Residual & 1.01435865 & 330 & .00307 & 814 & & R-squared & 0.1924 \\
\hline & & & & & & \multirow{2}{*}{$\begin{array}{l}\text { Adj R-squared } \\
\text { Root MSE }\end{array}$} & 0.1826 \\
\hline Total & 1.25594613 & 334 & .0037 & 318 & & & .05544 \\
\hline ABS_P_98 & Coef. & Std. & Err. & $t$ & $\mathrm{P}>|\mathrm{t}|$ & [958 Conf. & Interval] \\
\hline GINIBANDAS & .201231 & .053 & 30097 & 3.80 & 0.000 & .0969514 & .3055107 \\
\hline ING_HOG_BASE97 & -.000012 & 6.12 & $2 e-06$ & -1.96 & 0.051 & -.0000241 & $3.05 e-08$ \\
\hline ING_MEDIO_CUAD & $6.04 e-11$ & 4.36 & $6 e-10$ & 0.14 & 0.890 & $-7.97 e-10$ & $9.17 e-10$ \\
\hline INDICE_URB & -.0357826 & .009 & 91347 & -3.92 & 0.000 & -.0537523 & -.017813 \\
\hline _cons & .3754347 & .022 & 28017 & 16.47 & 0.000 & .3305796 & .4202897 \\
\hline
\end{tabular}

Fuente: Stata y cálculos propios.

Encontramos para este año que la relación entre la participación electoral y el índice de Gini es directa y significativa. Sin embargo, surgió la duda de si la desigualdad influía sobre la participación o el efecto era inverso, en otras palabras, se consideró un problema de endogeneidad por los siguientes motivos:

1. Altos niveles de participación pueden reducir la desigualdad de ingresos al incrementar la disponibilidad de información, opciones y oportunidades. De este modo se sospecha la presencia de una causalidad inversa donde la participación explique la desigualdad.

2. Similarmente se puede considerar que aquellos municipios que demuestren mayores niveles de abstención, pueden ser objetivos de políticas redistributivas que reduzcan los niveles de desigualdad por parte de autoridades en búsqueda de votos. En ese sentido también se puede sospechar la presencia de un problema de causalidad inversa. 
3. Asimismo, puede existir un problema de valores omitidos no incorporados en el modelo que sobreestimen o subestimen la explicación de la variable dependiente. Un ejemplo puede ser la influencia de variables macroeconómicas como el PIB per cápita, el Gasto Fiscal y la Inflación -registrado a nivel municipal- con los cuales no se contó y cuyos efectos pueden ser importantes incentivos -favorables o no- para ir a votar.

Para corregir este problema se tuvo que hacer una prueba de 2SLS o mínimos cuadrados en dos etapas (Espinoza, 2010). Se tomaron en consideración múltiples variables como posibles Variables Instrumentales. El instrumento que resultó válido y significativo fue la estructura poblacional activa por municipio (ESTRUC_POB_ACT), al comprobar su elevada significancia contra la variable independiente con una confianza del 1\% y su poca relación directa contra la variable explicada. Esto es presentado en la siguiente tabla:

\section{Tabla 2}

Primera etapa del modelo de Mínimos Cuadrados Ordinarios (MCO) para 1998.

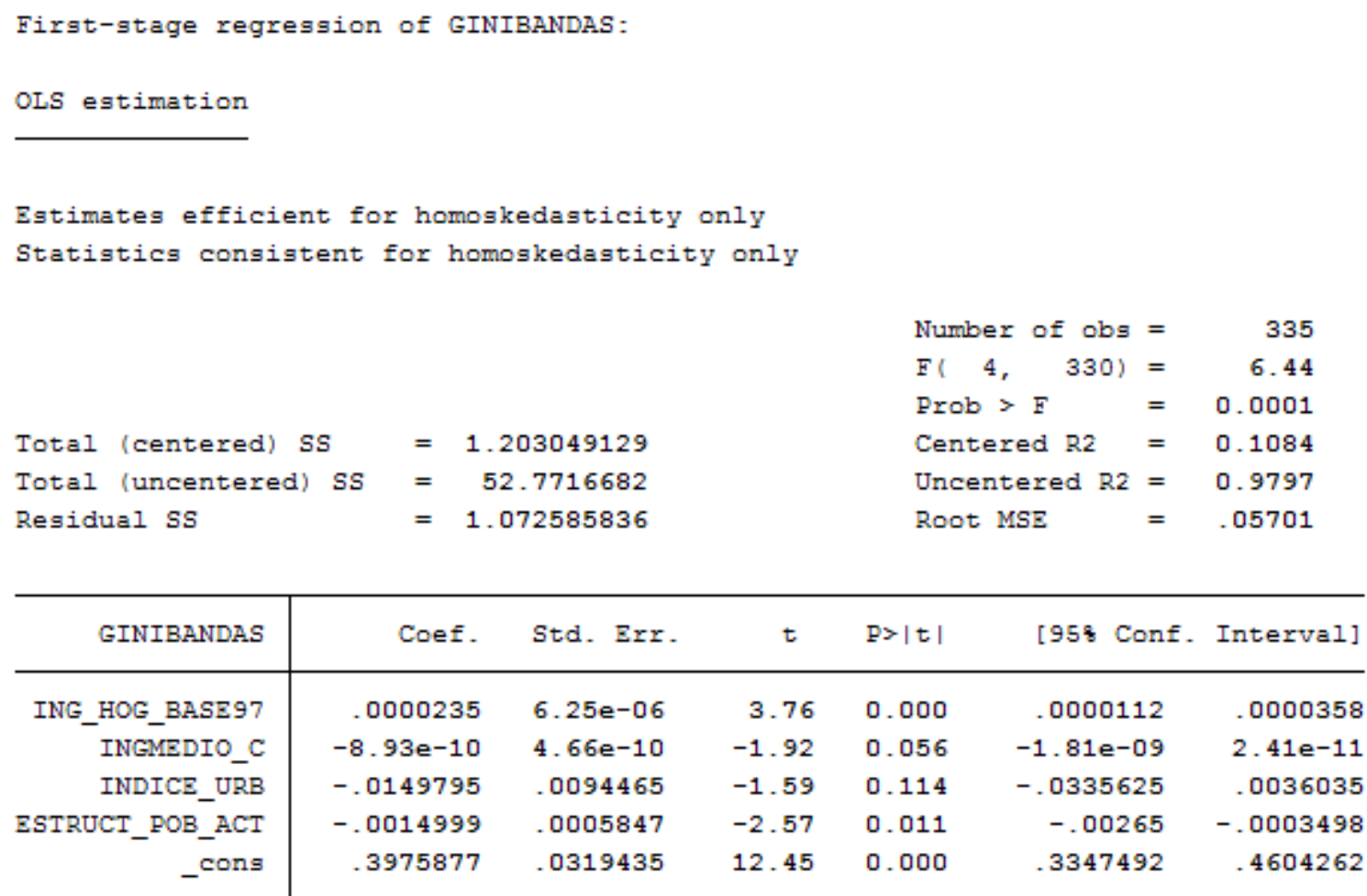

Included instruments: ING_HOG_BASE97 INGMEDIO_C INDICE_URB ESTRUCT_POB_ACT

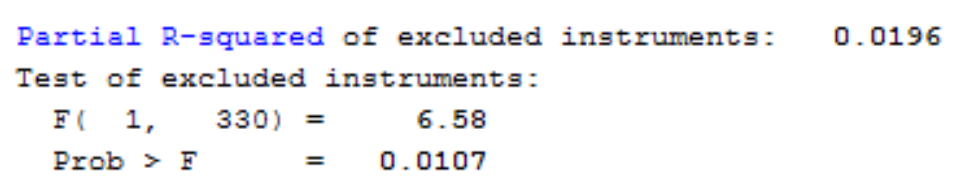

Fuente: Stata y cálculos propios.

Pérez López, Renato 
Apreciando la segunda etapa de la prueba -con ESTRUCT_POB_ACT-, se evidencia la significancia de la variable independiente al $10 \%$ de confianza, al igual que un incremento en su coeficiente a 0.8364 . El aumento drástico en el coeficiente de la variable independiente resultó similar al reportado por Alesina \& La Ferrara, pudiendo significar que el problema de endogeneidad presentado respondió a la presencia de causalidad inversa en la explicación del modelo. La variable dependiente pudo influir a su vez sobre la variable explicativa subestimando la influencia de la desigualdad de ingresos sobre la abstención.

La segunda etapa del modelo de Mínimos Cuadrados Ordinarios (MCO) se muestra a continuación:

\section{Tabla 3}

Segunda etapa del modelo de mínimos cuadrados ordinarios(MCO) para 1998

IV (2SLS) estimation

Estimates efficient for homoskedasticity only

Statistics consistent for homoskedasticity only

$\begin{array}{lllr} & & \text { Number of obs }= & 335 \\ & & \text { F }(4,330)= & 5.56 \\ & & \text { Prob }>\mathrm{F} & =0.002 \\ \text { Total (centered) SS } & =1.255946133 & \text { Centered R2 } & -0.1590 \\ \text { Total (uncentered) SS } & =50.95055115 & \text { Uncentered R2 } & 0.971 \\ \text { Residual SS } & =1.455689366 & \text { Root MSE } & .06592\end{array}$

\begin{tabular}{r|rrrrrr}
\hline ABS_P_98 & Coef. & Std. Err. & $\mathbf{z}$ & P $>|\mathbf{z}|$ & [958 Conf. Interval] \\
\hline GINIBANDAS & .8364143 & .4513262 & 1.85 & 0.064 & -.0481689 & 1.720997 \\
ING_HOG_BASE97 & -.000029 & .000014 & -2.07 & 0.038 & -.0000564 & $-1.58 e-06$ \\
ING_MEDIO_CUAD & $8.66 e-10$ & $7.68 e-10$ & 1.13 & 0.259 & $-6.39 e-10$ & $2.37 e-09$ \\
INDICE_URB & -.0239238 & .0136959 & -1.75 & 0.081 & -.0507672 & .0029196 \\
_COng & .168613 & .14802 & 1.14 & 0.255 & -.1215009 & .4587269 \\
\hline
\end{tabular}

Underidentification test (Anderson canon. corr. LM statistic) : 125.490

Chi-sq(1) P-val $=0.0000$

Weak identification test (Cragg-Donald Wald F statistic):

197.661

Stock-Yogo weak ID test critical values: 108 maximal IV size

16.38

158 maximal IV size

8.96

208 maximal IV size

6.66

258 maximal IV size

5.53

Source: Stock-Yogo (2005). Reproduced by permission.

Fuente: Stata y cálculos propios.

REVISTA DE INVESTIGACIÓN SIGMA / Vol. 06, № 1, 2019 (pág. 50-66) 
Para ratificar la corrección del problema de endogeneidad que permitió el instrumento utilizado, se corrió el comando ivendog resultando que:

\section{Tabla 4}

Prueba de endogeneidad del modelo para 1998.

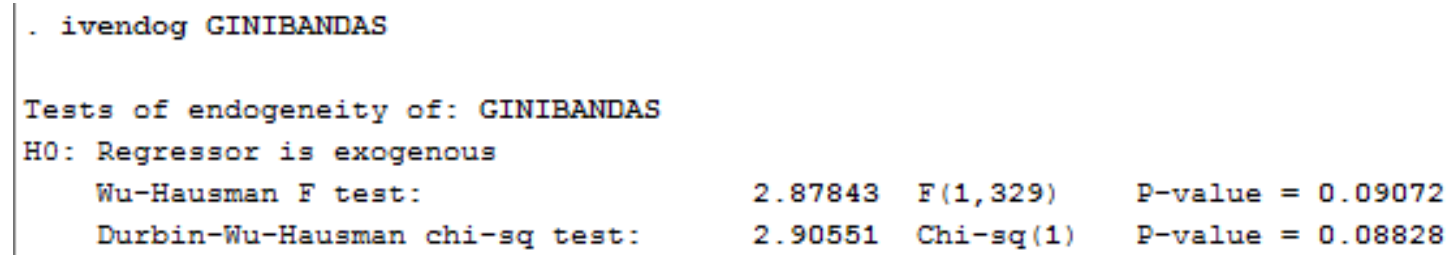

Fuente: Stata y cálculos propios.

Con una significancia del $9 \%$ se acepta observacional encontrada, sugiriendo la hipótesis nula, que el regresor es exógeno, mecanismos de causalidad inversa que reducen en otras palabras, se pudo corregir el problema los estimados brutos. Con ello se entiende que de endogeneidad. El resultado demuestra pudo haber un efecto negativo de la participación contundentemente que mayores niveles de electoral sobre los niveles de desigualdad social abstención en el año 1998 se asocian con que logró corregirse. mayores niveles de desigualdad intramunicipal observados en el año 2001; donde el estimado del efecto causal es mayor que la asociación

El mismo estudio se hizo para el año 2001. La siguiente tabla muestra los resultados obtenidos para el modelo base:

\section{Tabla 5}

Modelo para 2012.

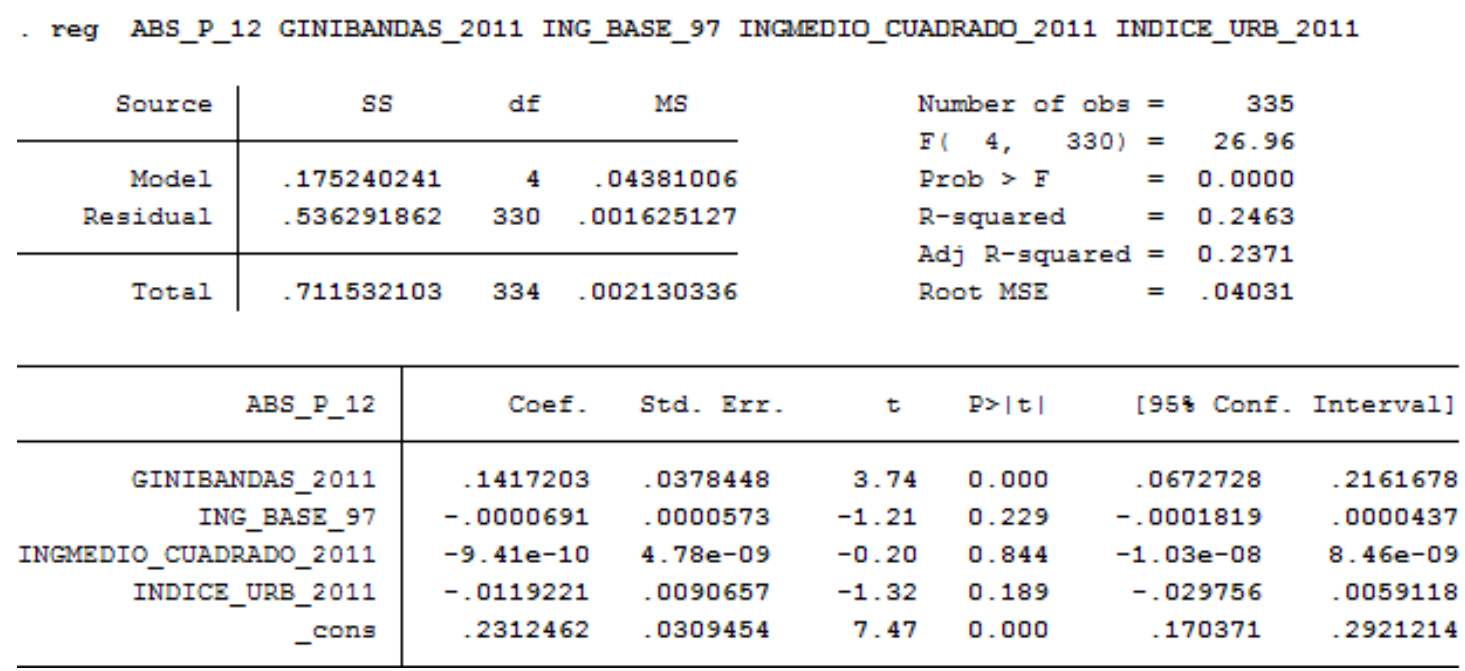

Fuente: Stata y cálculos propios.

Pérez López, Renato 
De igual manera, encontramos para este año que la relación entre la participación electoral y el índice de Gini es directa y significativa, entendiendo que mayores niveles de desigualdad de ingresos se asocian con mayores niveles de abstención. Sin embargo, se tomó en cuenta el problema de endogeneidad, por lo que se hicieron las pruebas pertinentes con la misma variable instrumental actualizada. Los resultados son presentados en la siguiente tabla.

\section{Tabla 6}

Primera etapa del modelo de Mínimos Cuadrados Ordinarios (MCO) para 2011.

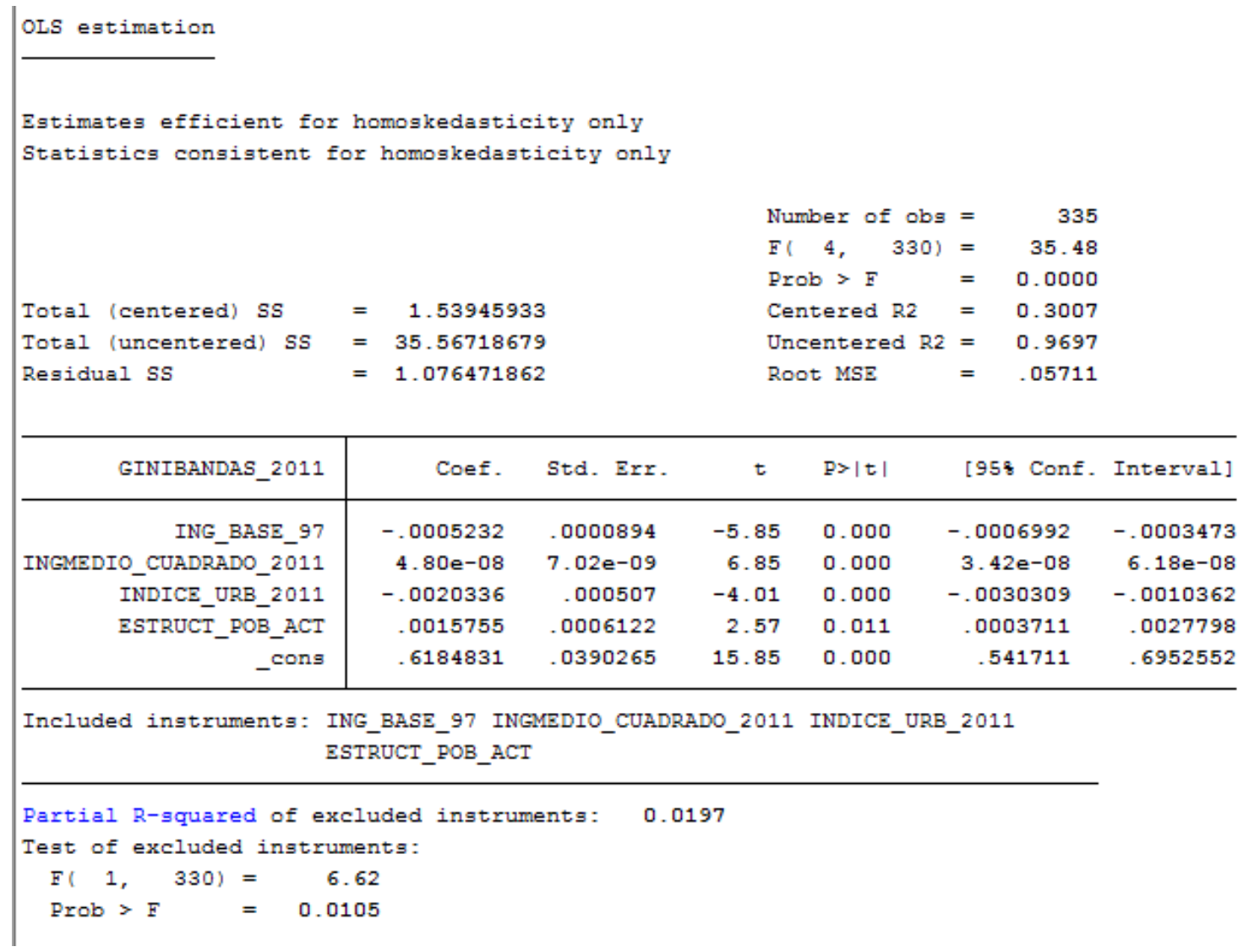

Fuente: Stata y cálculos propios.

Apreciando la segunda etapa de la prueba -con ESTRUCT_POB_ACT-, se evidencia la significancia de la variable independiente al $10 \%$ de confianza, al igual que un incremento en su coeficiente a 0.7149 . El aumento drástico en el coeficiente de la variable independiente resultó similar al reportado en el modelo anterior, pudiendo significar que el problema de endogeneidad presentado respondió a la presencia de causalidad inversa en la explicación del modelo. La variable dependiente pudo influir a su vez sobre la variable explicativa subestimando la influencia de la desigualdad de ingresos sobre la abstención.

La segunda etapa del modelo de Mínimos Cuadrados Ordinarios (MCO) se muestra a continuación: 


\section{Tabla 7}

Segunda etapa del modelo de Minimos Cuadrados Ordinarios (MCO) para 2011.

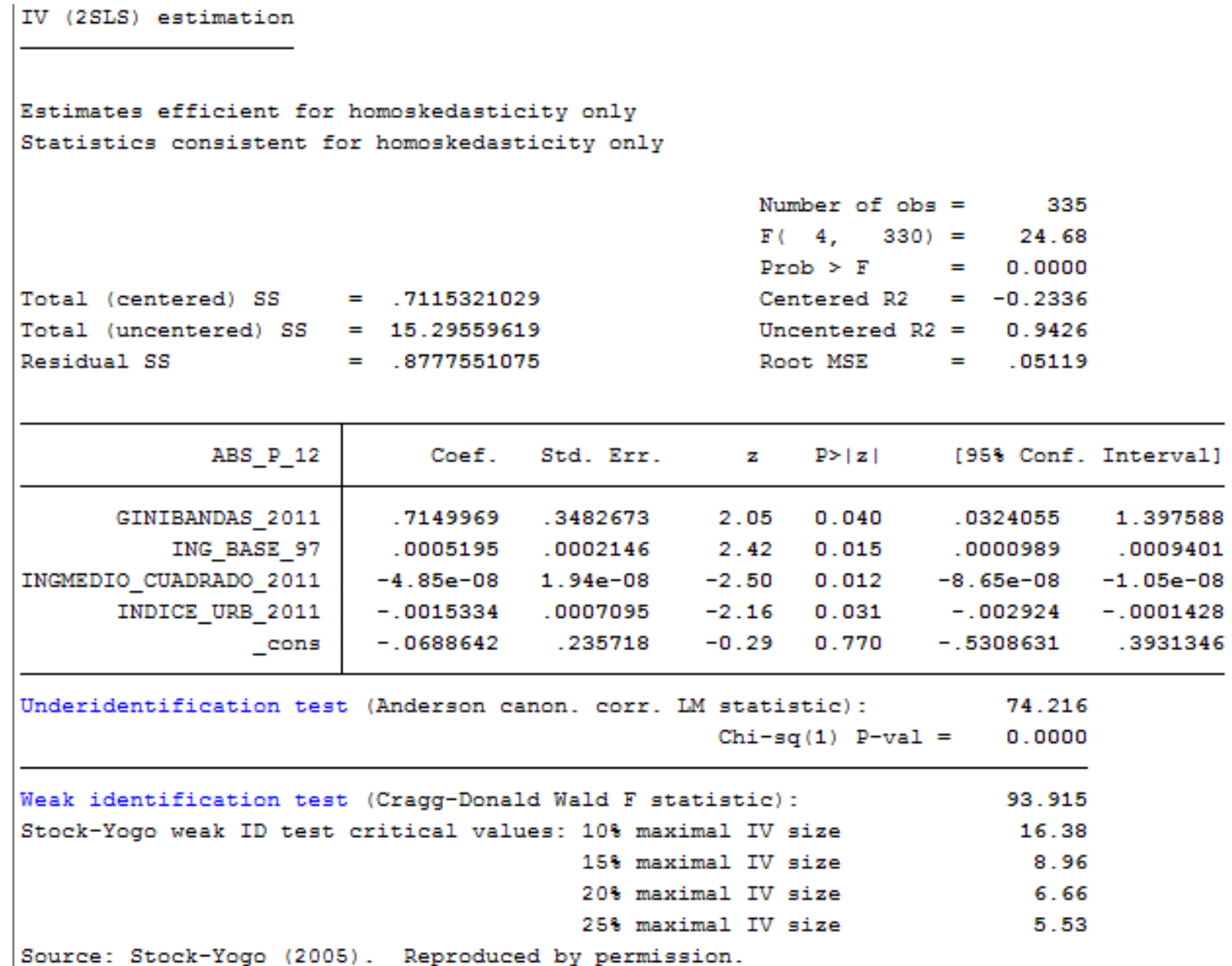

Fuente: Stata y cálculos propios.

Para ratificar la corrección del problema de endogeneidad que permitió el instrumento utilizado, se corrió el comando ivendog resultando que:

\section{Tabla 8}

Prueba de endogeneidad del modelo para 1998

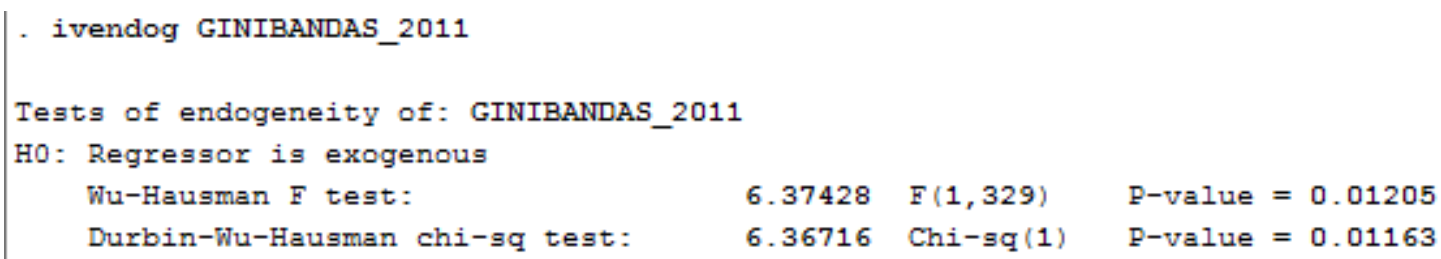

Fuente: Stata y cálculos propios.

Pérez López, Renato

La desigualdad de ingresos y su incidencia en la participación electoral venezolana 
Con una significancia del $1 \%$ se acepta la hipótesis nula, que el regresor es exógeno, en otras palabras, la variable instrumental pudo corregir el problema de endogeneidad del modelo.

Nuevamente, el resultado obtenido demuestra que mayores niveles de abstención en el año 2012 se asocian con mayores niveles de desigualdad en el año 2011. Resumirá los hallazgos más importantes, relacionando las propias observaciones con estudios de interés, señalando aportaciones y limitaciones, sin redundar datos ya comentados en otros apartados. La discusión de los resultados debe ser clara, concisa y contrastada con otros estudios.

\section{Conclusiones}

Analizados los resultados del modelo, el estudio demostró que en el caso venezolano, comunidades más heterogéneas tienden a ser menos participativas. El modelo utilizado resultó ser válido en la explicación entre ambas variables en dos momentos distintos del tiempo. Además, se pudo estimar la relación causal desigualdadparticipación mediante el uso de variables instrumentales. El provecho de un instrumento válido que corrigiera el problema de endogeneidad comprobado, llevó a concluir que la abstención pudo motivar políticas redistributivas para ganar votos por causalidad inversa.

Dehecho, conbase enlacalidad explicativa de la primera regresión construida en el trabajo, se pudo contrastar el comportamiento electoral de varios municipios bajo distintas condiciones de desigualdad y evaluar sus resultados. El ejercicio con el Municipio Libertador del Distrito Capital -cuya abstención se ubicó en el orden del
34,3\% para 1998- demostró que de tener, ceteris paribus, los niveles de desigualdad presentados en el año 2011 de 0,3724 puntos del índice de Gini -por debajo de sus 0,456 registrados en 2001- la abstención disminuiría en casi dos puntos porcentuales, elevando la participación en treinta y cuatro mil trescientas cuarenta y siete (34.347) personas, en otras palabras, aumentaría la participación en $1,87 \%$.

Por otro lado, un $13 \%$ de los municipios presentaron comportamientos atípicos, dado que presentaron mayores niveles de desigualdad para el año 2011 respecto a 2001 y sus niveles de participación aumentaron, contradiciendo la hipótesis del trabajo. Se puede intuir que estos resultados se deben a una baja declaración de ingresos al momento de llevarse a cabo el censo, pues estos sitios son principalmente municipios rurales con bajos niveles de población, por lo que, si algunas familias, 10 por ejemplo, dejan de declarar sus ingresos se generarían sesgos en el índice de Gini de estas zonas. Algunos de estos casos son: los municipios Manapiare, Alto Orinoco en Amazonas, Antonio Díaz en Delta Amacuro, entre otros.

Siguiendo los resultados obtenidos, se esperaría que los niveles de participación en Venezuela continúen aumentando en el tiempo, dada la tendencia en la disminución de los niveles de desigualdad de ingresos para el período 20012011.

Adicional a la lectura del fenómeno causal que representa la desigualdad social sobre la participación ciudadana en los municipios venezolanos, se pudo interpretar cómo las fuerzas de los distintos actores de la sociedad 
involucrados interactúan en torno a ellas. Por un lado, los partidos políticos, que no logran vincularse suficientemente con sus electores, afectando ello la deseada efectividad de lograr identidades y propuestas de gobierno que motiven la participación y el activismo del ciudadano. Por otra parte, en la sociedad civil escasean incentivos para reunirse, interactuar y organizarse en grupos por falta de contundencia en los intereses comunes que fortalezcan el capital social. De igual forma el Estado se presenta con la tarea de dirigir políticas efectivas en satisfacer las necesidades de toda la población, por falta de homogeneidad en las problemáticas e intereses de cada clase.

En síntesis, la relevancia de descifrar cuales son los incentivos que desencadenan la participación ciudadana radica en su capacidad de fortalecer el capital social, las redes y el tejido socio-económico de un país, que combinado con una buena dotación de capital físico y humano, representa una fórmula para el éxito.

Venezuela es un buen ejemplo para explicar que los capitales físicos y humanos no son suficientes para generar el bienestar sostenido que caracteriza a los países desarrollados. Existen otras formas de capital de relevancia como lo es el capital social, el cual ha demostrado tener un rol catalítico sobre las grandes economías del mundo que merece ser objeto del mayor estudio. Este estudio puede servir como base para presentar y estudiar proyectos futuros de la mano de los hacedores de políticas del país, de manera de alimentar y buscar profundizar en la participación ciudadana en pro del desarrollo social y del capital humano.

\section{Referencias bibliográficas}

Alesina, A. \& La Ferrara, E. (2000). Participation In Heteregenous Communities. The Quarterly Journal of Economics, MIT Press, vol. 115(3), pp. 847-904.

Banfield, E. (1958). The Moral Basis of a Backward Society. Glencoe, IL: The Free Press.

Briceño, H. (2009). Participación Electoral y cultura política en Venezuela 1958-2007. Cuadernos del Cendes vol, $\mathrm{n}^{\mathrm{o}} 72$, pp. 3766.

Domènech, A. (22 de Mayo de 2011). Raíces de la abstención electoral. Blog diariolínea. Recuperado de http://diariolinea.blogspot. com/2011/05/raices-de-la-abstencionelectoral.html

Fukuyama, F. (1995). Social Capital and the Global Economy. Foreign Affairs, vol. 74, $\mathrm{n}^{\circ}$ 5, pp. 89-103.

Goerlich , F. (1998). Desigualdad, Diversidad y Convergencia: (algunos) instrumentos de medida. Recuperado de http://www.ivie.es/ downloads/docs/mono/mono1998-01.pdf

Kim, J., Verba, S., Norman, H. (1978). Participation and political equality: $A$ seven-nation comparison. Cambridge, Inglaterra. Cambridge University Press.

López-Aranguren, E. (2005). Problemas Sociales: Desigualdad, pobreza, exclusión social. Madrid, España. Editorial Biblioteca Nueva.

Pérez, C. (2006). Enfoques teóricosmetodológicos en el estudio de la 
participación electoral. Cuestiones

Politicas. vol. 22, n 37, pp.74-93.

Phelan, M. \& Fundación Escuela de Gerencia Social. (2006). La desigualdad en Venezuela. Ministerio del Poder Popular para la Planificación y el Desarrollo. Caracas.

Pickett K, \& Wilkinson, R. (2010). The Spirit Level: Why Equality is Better for Everyone. Penguin Books. 2da edición. 2010

Putnam, R. (1993). The Prosperous Community: Social Capital and Public Life. The American Prospect, $n^{o} 13$, pp.35-42.

Putnam, R. (2000). Bowling Alone: The Collapse and Revival of American Community. New York: Simon \& Schuster. 2000.

Salamanca, L. (2012). ¿Por qué vota la gente?, Caracas, Editorial Alfa.

Silva, M. (2010). Desigualdad y Exclusión Social: de breve revisitación a una síntesis proteóricarips.. Revista de Investigaciones Politicas y Sociológicas. Recuperado de http://www.redalyc.org/articulo. oa? id $=38015080007$

Theil, H. (1967). Economics and information Theory, Amsterdam, North-Holland. 